
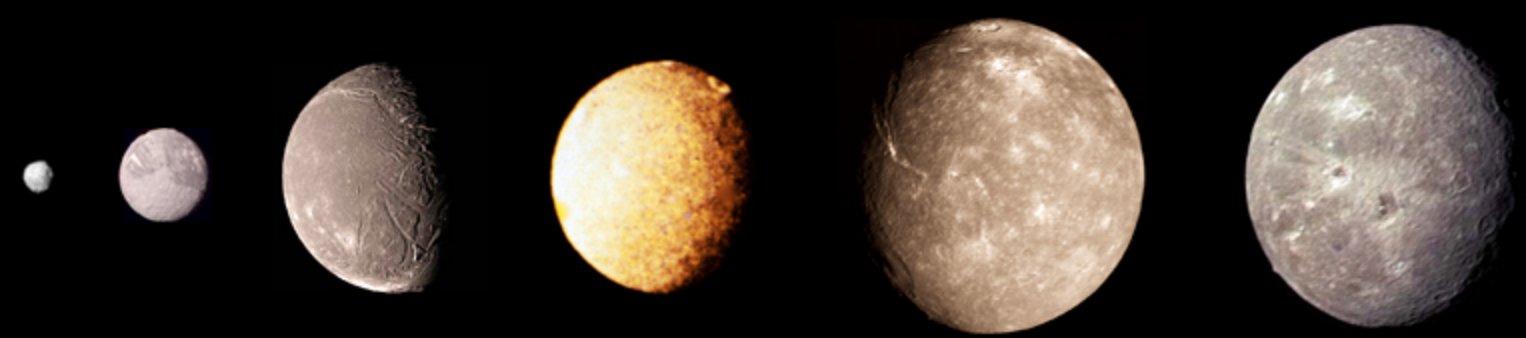

\title{
A New Frontiers Class Mission for the Uranian System that Focuses on Moon, Magnetosphere, and Ring Science
}

\author{
A White Paper in Response to the Planetary Science and Astrobiology Decadal Survey 2023-2032 Call
}

Erin Leonard ${ }^{\text {* }}$ (Erin.J.Leonard@ipl.nasa.gov; +1 626318 2617)

Coauthors: Catherine Elder ${ }^{1}$, Tom Nordheim ${ }^{1}$, Richard Cartwright ${ }^{2,3}$, D. Alex Patthoff ${ }^{4}$, Chloe Beddingfield $^{2,3}$, Matthew Tiscareno ${ }^{3}$, Nathan Strange ${ }^{1}$, and Tibor Balint ${ }^{1}$

Cosigners/Endorsers (98): Robert Pappalardo ${ }^{1}$, Corey Cochrane ${ }^{1}$, Imke de Pater ${ }^{5}$, Mike Sori ${ }^{6}$, Insoo Jun $^{1}$, Bonnie Buratti ${ }^{1}$, Adam Masters ${ }^{7}$, Hao Cao ${ }^{8}$, Julieanne Moses ${ }^{9}$, Chris Paranicus ${ }^{10}$, Alice Lucchetti ${ }^{11}$, Maurizio Pajola ${ }^{11}$, Caitlin Ahrens ${ }^{12}$, Michael Lucas ${ }^{13}$, William Grundy ${ }^{14}$, Douglas Hemingway ${ }^{15}$, Kevin Baillié $^{16}$, Karen Aplin ${ }^{17}$, Ian Cohen ${ }^{10}$, Stephanie Jarmak ${ }^{18}$, Shawn Brooks ${ }^{1}$, Marina Brozovic ${ }^{1}$, Francesca Scipioni $^{3}$, Rosaly Lopes ${ }^{1}$, Dale Cruikshank ${ }^{2}$, Tracy Becker ${ }^{18}$, Sierra Ferguson ${ }^{19}$, Emily Martin ${ }^{20}$, Xianzhe $\mathrm{Jia}^{21}$, Robert Marcialis ${ }^{22}$, David DeColibus ${ }^{23}$, Andrew R. Poppe ${ }^{5}$, Thomas Roatsch ${ }^{24}$, Andrew Coates ${ }^{25}$, Chuanfei Dong ${ }^{26}$, Louise Prockter ${ }^{27}$, Wes Patterson ${ }^{10}$, Gina DiBraccio ${ }^{28}$, James Roberts ${ }^{10}$, Frank Postberg $^{29}$, Samuel Howell ${ }^{1}$, Hsiang-Wen Hsu ${ }^{30}$, Scott Sheppard ${ }^{15}$, Carol Paty ${ }^{31}$, Hamish Hay ${ }^{1}$, Benjamin Weiss $^{32}$, Jessica Mink ${ }^{8}$, Amy C. Barr ${ }^{4}$, Jamie Jasinski ${ }^{1}$, Leonardo H. Regoli ${ }^{10}$, Baptiste Journaux ${ }^{33}$, Ellen C. Czaplinski ${ }^{12}$, Marissa Cameron ${ }^{1}$, Devon Burr ${ }^{34}$, Tilmann Denk ${ }^{24}$, Joseph Lazio ${ }^{1}$, Paolo Tortora ${ }^{35}$, Sebastien Rodriguez $^{36}$, George Hospodarsky ${ }^{37}$, Ashley Schoenfeld ${ }^{38}$, Ingrid Daubar ${ }^{39}$, Margaret Kivelson $^{38}$, Jared Espley ${ }^{28}$, Elizabeth Turtle ${ }^{10}$, Kimberly Moore ${ }^{40}$, Emma Dahl ${ }^{23}$, Marshall J. Styczinski ${ }^{33}$, Emily Hawkins $^{41}$, Foivos Karakostos ${ }^{42}$, Lynnae Quick ${ }^{28}$, Yasmina Martos ${ }^{28}$, Simon Kattenhorn ${ }^{43}$, David Senske $^{1}$, Matthew Hedman ${ }^{44}$, James T. Keane ${ }^{1}$, Maheenuz Zaman ${ }^{45}$, Oliver White ${ }^{3}$, Oleg Shebanits ${ }^{7}$, C. Adeene Denton ${ }^{6}$, Michael Malaska ${ }^{1}$, Kelsi Singer ${ }^{18}$, Michelle Kirchoff ${ }^{18}$, Noah Hammond ${ }^{46}$, Jennifer Scully $^{1}$, Katherine de Kleer ${ }^{40}$, Michaela Villarreal ${ }^{1}$, Alyssa Rhoden ${ }^{18}$, Paul Byrne ${ }^{47}$, Robert W. Ebert ${ }^{18}$, Lauren Schurmeier $^{48}$, Linda Spilker ${ }^{1}$, Jessica Noviello ${ }^{19}$, Peter Kollmann ${ }^{10}$, Racine Swick ${ }^{12}$, Paul Helfenstein $^{49}$, Mark Neveu ${ }^{28}$, Geoff Collins ${ }^{46}$, Mallory Kinczyk ${ }^{47}$

\footnotetext{
${ }^{1}$ Jet Propulsion Laboratory, California Institute of Technology, ${ }^{2}$ NASA Ames Research Center, ${ }^{3}$ SETI Institute, ${ }^{4}$ Planetary Science Institute, ${ }^{5}$ University of California Berkeley, ${ }^{6}$ Purdue University, ${ }^{7}$ Imperial College London, ${ }^{8}$ Harvard University, ${ }^{9}$ Space Science Institute, ${ }^{10}$ Applied Physics Laboratory, John Hopkins University, ${ }^{11}$ INAF-OAPD Astronomical Observatory of Padova, ${ }^{12}$ University of Arkansas, ${ }^{13}$ University of Tennessee, ${ }^{14}$ Lowell Observatory, ${ }^{15}$ Carnegie Institution for Science, ${ }^{16} \mathrm{IMCCE}$, Observatoire de Paris, CNRS, ${ }^{17}$ University of Bristol, ${ }^{18}$ Southwest Research Institute, ${ }^{19}$ Arizona State University, ${ }^{20}$ Smithsonian Institute, ${ }^{21}$ University of Michigan, ${ }^{22}$ University of Arizona, ${ }^{23}$ New Mexico State University, ${ }^{24}$ German Aerospace Center (DLR), ${ }^{25}$ University College London, ${ }^{26}$ Princeton University, ${ }^{27}$ Lunar Planetary Institute, ${ }^{28}$ NASA Goddard Space Flight Center, ${ }^{29}$ Freie University of Berlin, ${ }^{30}$ University of Colorado Boulder, ${ }^{31}$ University of Oregon, ${ }^{32}$ Massachusets Institute of Technology,

${ }^{33}$ University of Washington, Seattle, ${ }^{34}$ Northern Arizona University, ${ }^{35}$ University of Bologna, Italy, ${ }^{36}$ IPGP, ${ }^{37}$ University of Iowa, ${ }^{38}$ University of California Los Angeles, ${ }^{39}$ Brown University, ${ }^{40}$ California Institute of Technology, ${ }^{41}$ Loyola Marymount University, ${ }^{42}$ University of Maryland, College Park, ${ }^{43}$ University of Alaska, Fairbanks, ${ }^{44}$ University of Idaho, ${ }^{45}$ Lamont-Doherty Earth Observatory, Columbia University, ${ }^{46}$ Wheaton College, ${ }^{47}$ North Carolina State University, ${ }^{48}$ University of Hawaii at Manoa, ${ }^{49}$ Cornell University
}

A portion of this research was carried out at the Jet Propulsion Laboratory, California Institute of Technology, under a contract with the National Aeronautics and Space Administration (80NM0018D0004). 


\section{Introduction}

The Uranian system is home to the best example of a native ice giant satellite system, a magnetosphere with a unique geometry and interaction with the solar wind, and a set of curiously narrow and dark rings. By investigating the major moons of Uranus as potential ocean worlds we would further our understanding of how ocean worlds originate and evolve throughout the solar system. The investigation of an ice giant magnetosphere would allow for new insights into fundamental magnetospheric physics as well as planetary dynamo theory. The investigation of the fascinating Uranian rings would provide insight into the origin and history of solid material around ice giants and the processes that operate in astrophysical disks. These three subsystemsthe moons, magnetosphere, and rings - are intricately coupled and could be investigated by an orbiting spacecraft with a small payload of high TRL instruments. Such a payload would include a camera, a mapping spectrometer, a magnetometer, a plasma spectrometer, and an energetic particle detector. It is critical for such a mission to arrive at the Uranian system by the early 2040s when the northern high-latitudes of the satellites (unimaged by Voyager 2), would still be visible. This opportunity will disappear once the system goes into southern spring in the late 2040s, not returning again until the 2090s.

The Uranian satellites offer our Solar System's best opportunity to study the origin and evolution of ice-giant satellites because Neptune's largest satellite Triton is likely a captured Kuiper Belt Object, which catastrophically disrupted Neptune's native satellite system ${ }^{1}$. The five largest Uranian satellites-Miranda, Ariel, Umbriel, Titania, and Oberon - exhibit tantalizing evidence of recent geologic activity ${ }^{2-6}$, suggesting these moons could host subsurface oceans now or in the recent past. The orbits of the rings and most of the Uranian moons reside within the Uranian magnetosphere and their surfaces are therefore continuously altered by magnetospheric plasma and energetic particles. Uranus's magnetosphere offers a unique laboratory to study the effects of a tilted dipole on solar wind-magnetosphere interactions that may be analogous to Earth's magnetosphere during polarity reversals ${ }^{7}$. Studying ice giant ring dynamics is a key investigative tool for understanding planet formation in planetary disks ${ }^{8,9}$.

A Flagship mission to Uranus would be needed for a comprehensive study of the entire system $^{10}$ and an ice giant Flagship mission has been endorsed by OPAG ${ }^{11}$. However, we propose that adding a Uranus system mission to the New Frontiers list is also needed to provide additional options for missions within the next decade to this understudied system. A New Frontiers class Uranus orbiter equipped with a camera, a visible and near-infrared mapping spectrometer, a magnetometer, a plasma spectrometer, and an energetic particle detector could return a wealth of data addressing three main science goals:

\section{Determine whether the Uranian satellites host subsurface oceans, search for signs of ongoing endogenic activity, and determine to what extent the surfaces are modified by exogenic processes.}

\section{Constrain the formation and evolution of the Uranian rings and their interactions with the major satellites.}

\section{Characterize the structure and dynamics of the Uranian magnetosphere,} including interactions with the solar wind and the Uranian moons and rings.

A mission with these synergistic science goals and a small payload could address additional high-priority questions about Uranus itself. Although a New Frontiers mission, particularly one

to the outer solar system, must have very focused objectives, the proposed payload could address complementary Uranus science (Section 2.4) including cloud tracking for atmospheric dynamics, remote composition, and ring seismology or gravity measurements for interior structure. 


\section{Uranian System Science}

A mission focusing on the Uranian moons, magnetosphere and rings would greatly contribute to our understanding on the formation and evolution of: ocean worlds ${ }^{12}$, proto-planetary disks ${ }^{8,9}$, ring systems around giant planets ${ }^{13}$, and non-dipolar or offset magnetospheres (for more details on ice giant magnetospheres, see Kollmann et al., 2020 $0^{14}$, a white paper for this decadal survey).

\subsection{Major Satellites}

Voyager 2 imaged the southern hemispheres of Uranus's five major satellites (diameters ranging from $472 \mathrm{~km}$ to $1578 \mathrm{~km}$; for further details on the major Uranian moons, see two other white papers for this decadal Cartwright and Beddingfield, 202015; Ahrens, 202016). Even with limited spatial coverage and generally low spatial resolution, those images show a geologically diverse set of satellites, with surfaces covered in fractures, ridges, altered craters, and other hints of recent activity ${ }^{2-6}$. Miranda and Ariel, the two smaller and better imaged worlds (highest resolution images at $\sim 240 \mathrm{~m} /$ pix and $\sim 1 \mathrm{~km} /$ pixel, respectively), display surfaces with complex tectonic features. Both show regions that may have formed through recent tectonic and/or cryovolcanic activity ${ }^{3-5,17}$ and have few impact craters (at the resolution limit). Some portions of Miranda appear older and more heavily cratered, a dichotomy akin to Saturn's active world Enceladus ${ }^{18}$. The other major moons were imaged at worse than $\sim 5 \mathrm{~km} /$ pixel by Voyager 2 , but even these low-resolution images hint at a resurfacing event on Umbriel and late-stage tectonics on Titania and Oberon ${ }^{2}$. All five major moons display evidence for ammonia-bearing species and carbon dioxide ice, volatiles that are not stable over geologic timescales ${ }^{19-21}$ suggesting recent activity such as cryovolcanism or tectonic activity ${ }^{6,22-25}$. Were these moons recently active, are they still active today, or would global high-resolution imagery suggest older features?
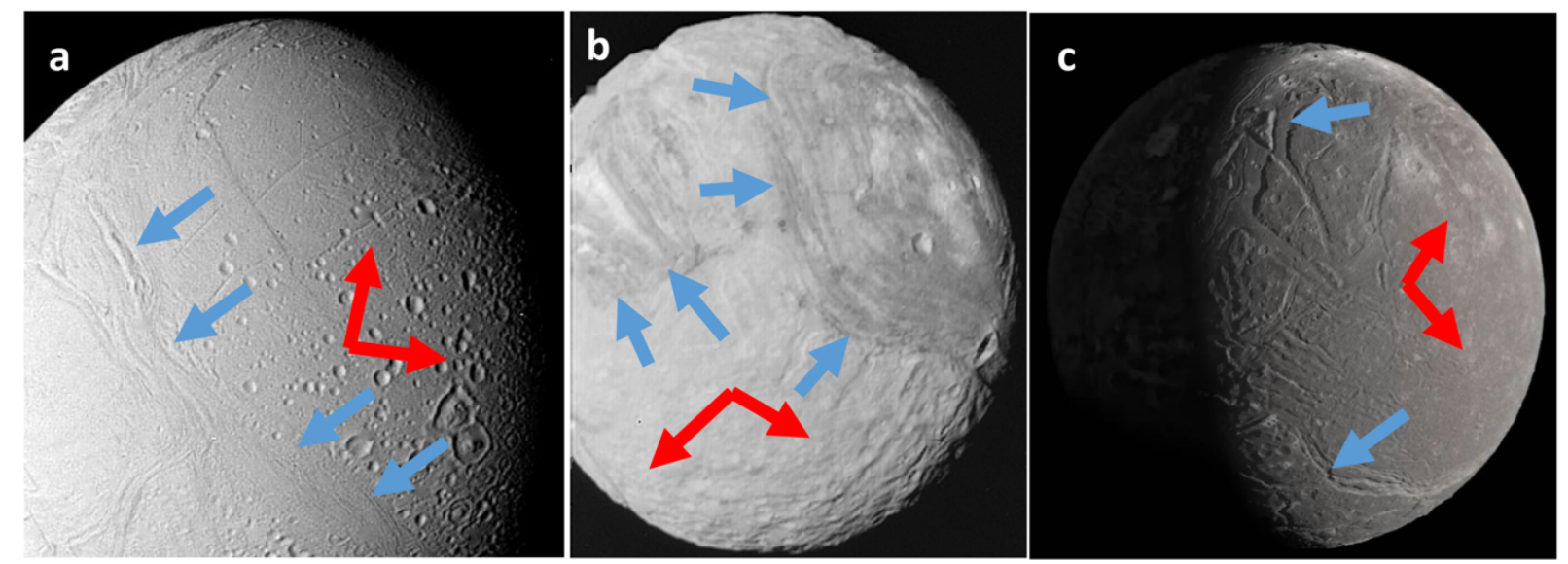

Figure 1:Voyager 2 image mosaics of (a) Enceladus $(d=504 \mathrm{~km})$, (b) Miranda $(d=472 \mathrm{~km})$, and (c) Ariel $(\mathrm{d}=1158$ $\mathrm{km}$ ) including night-side illumination by Uranus shine (Styrk and Stooke, 2008). Cratered regions (red arrows) and boundaries of isolated resurfacing (blue arrows) highlighted in each figure. Miranda and Ariel show evidence of partial resurfacing, analogous to Enceladus. Image credit: NASA/JPL/Caltech/USGS.

Furthermore, recent dynamical modeling work suggests that a 5:3 mean motion resonance, shared between Ariel and Umbriel in the recent past, may have resulted in sufficient tidal heating of Miranda to form the young Arden and Inverness coronae ${ }^{26}$. These indirect lines of evidencepresence of volatiles $^{19-21,27}$, geologically young terrain ${ }^{6,22-25}$, and potential dynamical history ${ }^{26}$ raise the question: Do any of the major Uranian moons host subsurface oceans today? Even if these moons are not ocean worlds, it would be a critical step to understanding how and under what conditions ocean worlds form ${ }^{12}$. 


\subsection{Rings and Minor Satellites}

Planetary rings are a natural laboratory in which we can study the dynamics analogous to protoplanetary disks ${ }^{8,9}$. Uranus's system of dark, dense and sharp-edged, narrow rings is unique among the giant planets ${ }^{28}$, constituting a third paradigm in contrast to the broad disk of Saturn's ice-rich rings and the more tenuous dusty rings of Jupiter and Neptune. Although the mechanism by which "shepherding" moons maintain the edges of the outermost $\varepsilon$ ring is well established ${ }^{29,30}$, the other sharp rings lack an observed shepherd. Thus, there is currently no valid self-sustaining model for narrow sharp-edged rings which, in addition to Uranus, would apply to the narrow rings of centaur Chariklo ${ }^{31}$ and dwarf planet Haumea ${ }^{32}$. Observations of Uranus' rings, even over a mission lifetime of $\sim 3$ years, would help constrain the formation of this dynamic system.

Additionally, the evolution of satellites and rings has become an increasingly controversial topic due to recent dynamical modeling suggesting that some of the Saturnian moons may have formed only $\sim 100 \mathrm{Ma}^{33}$ ago, whereas the geologic evidence of heavily cratered satellite surfaces points to an early formation in the Saturnian system ${ }^{34}$. Do the present-day rings of Uranus and their interactions with the Uranian satellites support early or more recent formation of the satellite and ring systems?

The composition of the dark Uranian rings is not known, as Voyager did not measure their composition and Earth-based telescopes have only obtained spectral measurements with a lack of diagnostic features ${ }^{35}$. The particle size distribution is also poorly known, and measuring it would enable comparisons to the rich structure found at Saturn ${ }^{36,37}$. Identifying the composition and particle size distribution of the Uranian rings would provide constraints on the history and evolution of the entire Uranus system.

The Uranian rings contain abundant km-scale structure, both radial and azimuthal, but much of it is uncharacterized and unexplained ${ }^{38,39}$. Furthermore, the Uranian rings could display oscillations that would allow for investigations of the interior structure of Uranus ('ring seismology'), similar to those that have led to advances in our understanding of the Saturnian interior $^{40}$. In addition to the relatively dense, sharp-edged rings, Uranus has dusty rings both inward and outward of the main rings, as well as interspersed within them. Dust must be continually replenished to maintain these rings ${ }^{41}$ and, while some sources of dust are known, many remain mysterious. Perhaps most puzzling, the $\mu$ ring is blue in color, implying a small and uniform grain size, with the orbit of the embedded ring moon Mab coinciding with the peak particle density of the $\mu$ ring $^{42,43}$. Endogenic geological activity on a moon as small as Mab (radius 6-12 km) is improbable, therefore: What is the origin of the $\boldsymbol{\mu}$ ring?

Observations of Mab and the other ring moons would be highly desired science enhancements, and possible with only little additional effort. Additionally, a Uranus orbiter could spend time looking outward to investigate the small and distant irregular satellites (including observation geometries never achievable from Earth), as was done with Cassini at Saturn $^{44}$. Observations of the irregular satellites would provide new knowledge on the origins of these likely captured objects, which in turn could reveal new insight into the dynamical evolution of small icy bodies in the outer Solar System ${ }^{45}$.

\subsection{Magnetosphere}

Uranus possesses a strong intrinsic magnetic field that has a surface field strength $\sim 2 / 3$ that of the Earth ${ }^{46}$. However, the dipole is offset from the center of Uranus by $\sim 0.3 \mathrm{R}_{\text {Uranus, }}$ and at the surface of Uranus, the dipole, quadrupole and octupole magnetic field components are about equal ${ }^{46-48}$. This indicates that the Uranian magnetic field may not be generated by a traditional 
dynamo ${ }^{49,50}$, but instead produced from currents in an electrically conductive "ice ocean" possibly consisting of superionic water ${ }^{51-53}$ (for additional details see Kollmann et al., 2020 $0^{14}$ ). Thus, by characterizing the Uranian magnetic field, we could infer properties of the internal magnetic field sources, as well as put important constraints on the internal structure and structural evolution of Uranus itself ${ }^{54}$. It is also important to note that such studies may also have implications for our understanding of Neptune, which has a similarly complex magnetic field, and extrasolar planets - whose magnetic fields, and thus potentially aspects of their interior conditions, may be inferred by remote observations from Earth.

The combination of Uranus's large obliquity of $97.9^{\circ}$ and its highly tilted $\left(59^{\circ}\right)$ and offset ( $\sim 0.3$ Ruranus) magnetic field leads to a configuration where the magnetospheric interaction with the solar wind varies considerably over both diurnal and seasonal timescales and may be reconfigured on timescales of less than a planetary rotation period ${ }^{55,56}$. At Uranian solstice, the magnetosphere takes on a configuration where one of the magnetic poles faces directly into the solar wind and the Uranian magnetotail takes on a twisted configuration not known to exist elsewhere in the solar system ${ }^{57}$. Is the configuration of the Uranian magnetosphere highly variable as predicted by models? Understanding the complicated flow of mass and energy through the system would inform how plasma and energetic particles interact with the satellites and rings. Understanding how the solar wind interacts with the Uranian magnetosphere would also help us understand past interactions between the solar wind with Earth's ancient paleomagnetosphere. For example, paleomagnetic measurements and dynamo models of the Earth suggest that the tilt of Earth's dipole could have exceeded $45^{\circ}$ during polarity reversals ${ }^{58}$.

Voyager 2 plasma measurements in the inner plasma sheet indicated that the solar wind is not an important source of magnetospheric plasma at Uranus, although intense substorm activity seems to indicate that there is a significant energy input from the solar wind ${ }^{59}$. However, Voyager 2 sampled only the inner plasma sheet, so it is not known whether the outer plasma sheet is dominated by solar wind plasma and to what extent the solar wind drives magnetospheric composition and dynamics at Uranus. To what extent is the Uranian magnetosphere driven by the solar wind versus internal processes?

\subsection{Uranus}

While the Uranian moons, magnetosphere and rings are a natural combination for a focused medium-cost mission, valuable Uranus science could also be achieved without significant added cost or mass to the mission design through use of the same instruments that would study the moons, magnetosphere, and rings. For example, cloud tracking would lead to important insights on atmospheric dynamics. Additionally, constraints on the interior structure of Uranus could be provided by measurements already accomplished by a moons, rings, and magnetosphere focused mission including: (1) gravity measurements on the close flybys of Uranus that are necessary to the tour design (see Section 3.5), (2) potential ring seismology (as done at Saturn ${ }^{40,60}$ ), and (3) measuring the structure and dynamics of the magnetic field (Section 2.3). Constraints on the interior structure of Uranus provide crucial information on the formation and evolution of the planet. In the case of the example mission concept described below, preliminary study indicates that an external contribution (e.g., a probe) could fit on the orbiter and depending on the capabilities, could add even more Uranus-focused science return.

\section{Example Mission Concept}

The Ice Giants Pre-Decadal Survey Mission Report ${ }^{54}$ considered flagship-class missions to the Uranian system. However, two recent concepts have shown that a New Frontiers class 
Uranus orbiter is possible, although both of these concepts required a narrow focus and did not include objectives relating to the moons or rings ${ }^{61,62}$ (for more discussion on potential New Frontiers class missions to the Uranian system see Cohen et al., 202063). The Uranus Magnetosphere and Moons Investigator (UMaMI) is an example New Frontiers class mission concept to study the Uranian magnetosphere, moons, and rings ${ }^{64}$. The measurements could be achieved with a high-resolution camera with color filters, a magnetometer, a visible and infrared (VIR) spectrometer, a plasma spectrometer, and an energetic particle detector. Suitable versions of each of these instruments have flown on previous missions making this a low-risk payload.

\subsection{Geology and composition of the major satellites}

The cameras that flew on the New Horizons mission would be capable of resolving satellite structures at the sub-km scale, revealing relationships between surface structures and heat flux, ice shell thickness, and ice rheology. The northern hemispheres should be particularly high priority as no imagery exists of those regions of the moons. Flybys of the moons would also reveal the distribution and spectral signature of surface constituents, as well as possible spatial association with geologic landforms. The Dawn Visible and Infrared (VIR) Mapping Spectrometer is likely a sufficient instrument for this critical task.

\subsection{Rings}

Images and compositional measurements (see instrument descriptions in Section 3.1) would greatly improve our understanding of the Uranian rings. High resolution imaging would reveal dynamical processes within the rings, providing insight into how the dense and narrow rings are maintained. Imaging the dusty rings over the mission lifetime ( $\sim 3$ years) and at a range of phase angles would shed light on their changing structures and the source of the dust. Characterizing Mab with high resolution imaging and spectral information may reveal if this small moon is the source of the $\mu$ ring, and how Mab is able to sustain it. Observations with NIR spectroscopy would reveal the composition and particle-size distribution of Uranus's rings, improving our understanding of the rings' origin and possible modification of the Uranian satellites' surface compositions. Optical depth profiles generated by the observation of occultations of stars by the rings would allow for a detailed characterization of ring structure.

\subsection{Magnetosphere}

Outstanding questions on Uranus's magnetosphere could be addressed by a suite of three high TRL instruments with strong flight heritage: a magnetometer (MAG), a plasma spectrometer (PS), and an energetic particle detector (EPD). MAG would measure threedimensional magnetic fields using three fluxgate sensors. The PS would measure energy, composition, and spatial distributions of plasma electrons and ions. EPD would measure the energy, composition and spatial distributions of energetic ions and electrons. By characterizing the Uranian magnetic field, magnetospheric plasma, and trapped energetic particles, we would be able to study the interaction of the solar wind with the Uranus system, and to determine to what extent the magnetosphere is driven by internal versus external processes. By searching for magnetic field signatures, energetic charged particle absorption signatures and pick-up ions, we would search for exospheres and outgassing activity at the major Uranian moons.

\subsubsection{Detecting subsurface oceans}

Magnetometer measurements of magnetic induction have revealed subsurface oceans in Europa and Callisto ${ }^{65,66}$. The magnetic moment of Saturn is nearly co-aligned with its spin axis, so this technique could not be employed for the saturnian satellites. In contrast, Uranus has a large magnetic moment that is tilted $59^{\circ}$ relative to its spin axis and the orbital axes of the moons ${ }^{48}$. 
Therefore, in the reference frame of the moons, the magnetic field varies in time and is synchronized with the perceived rotation of Uranus and the orbital position of the moon. An initial assessment of Uranus's field at the position of Miranda and Ariel indicates that both are prime targets for magnetic induction studies. If salty subsurface oceans are present within these moons, the signatures of the induced field would be large and readily detectable during moon flybys.

\subsection{Mission Design Feasibility and Viability}

Two recent studies strengthen the feasibility of a Uranus orbiter fitting within New Frontiers. A potential design for a Uranus orbiter tour with a small $400 \mathrm{~kg}$ small spacecraft was demonstrated in Conversano et al. (2019) ${ }^{67}$. Similar trajectories could be readily scaled to larger spacecraft, and even an Atlas V-551 or Delta IV Heavy class launch vehicle could deliver 1000 to $1900 \mathrm{~kg}$ useful mass into orbit after Uranus Orbit Insertion, which is in family with selected New Frontiers class missions. Allowing for an 11-year flight time and at least 3 years for a Uranus system tour, this mission design falls within the typical design lifetime of a Radioisotope Power System of $\sim 14$ years. A three-year Uranian tour could allow multiple low and slow $(<100$ $\mathrm{km},<1 \mathrm{~km} / \mathrm{s}$ ) flybys of all of the major Uranian satellites, as well as broad sampling of the Uranian magnetosphere. In addition, low passes over Uranus (within one Uranus radii) over a range of latitudes could enable gravity science and atmospheric occultation investigations. The ring skimming technique developed by Vaquero et al. (2019) ${ }^{68}$ could be applied at Uranus to allow low $(<1000 \mathrm{~km})$ passes over the ring system and radio occultations of the rings.

A modest payload allocation of 50-100 kg could accommodate the payload described in this whitepaper. Leveraging recent developments for SmallSat-inspired instruments and subsystems could significantly reduce the mass and power requirements for this mission. (For more details, see Balint et al., 202069, a white paper for this decadal survey.) A preliminary cost estimate for a model payload was within the cost allocation for other implemented or proposed NF class missions. While these analogies and piecewise assessment showed initial feasibility, a subsequent higher maturity study is recommended for refinement.

As demonstrated in this report, the Uranian system requires drawing on perspectives spanning the gamut of planetary science, geoscience, astronomy, technology, engineering, and beyond. Thus, we recommend that the decadal survey consider the critical role of team dynamics, equity, diversity, inclusion, and accessibility in planetary science. Additionally, the majority of the authors and cosigners on this white paper are early-career, demonstrating interest and support from this critical group given the inherent timescales involved in ice giant missions

\section{Recommendation}

A mission focusing specifically on the complementary science of the Uranian moons, magnetosphere, and rings could be achieved within the New Frontiers cost-cap. Observations by such a mission would provide insight into: (1) the conditions that result in icy satellites becoming ocean worlds, (2) evolution of a narrow and chaotic ring system and protoplanetary formation, and (3) fundamental magnetospheric physics. Additionally, an atmospheric probe provided by a partner agency could enable additional Uranus science. Thus, this mission could aid in answering broader questions on the formation and evolution of the solar system and habitable environments therein. While there are other possible focuses for a New Frontiers class mission to the Uranian system, a focus on the moon, magnetosphere, and rings allows for affordable yet powerful overarching system science that has broad implications. Overall, we recommend that the Uranian system be added to the New Frontiers list to provide a wide range of options for missions to investigate the moons, magnetosphere, and rings of this understudied system. 


\section{References}

1. Agnor, C.B. and Hamilton, D.P., 2006. Nature, 441, 192.

2. Smith, B.A., et al., 1986. Science 233, 43-64.

3. Schenk, P. M., 1991. JGR, 96. 1887-1906.

4. Pappalardo, R.T., et al., 1997. JGR Planets

102.E6, 13369-13379.

5. Beddingfield, C.B, et al., 2015. Icarus 247, 35-52.

6. Leonard, E.J., and Patthoff, D.A., 2019. EPSC-

DPS Joint Meeting 2019.

7. Glatzmaiers, G.A. and Roberts, P.H., 1995.

Nature 377, 203-209.

8. Wyatt, Mark C. , 2008. Annu. Rev. Astron.

Astrophys. 46, 339-383.

9. Spilker, T.R. Acta Astronautica 52.2-6 (2003):

259-265.

10. Beddingfield et al. (2020) PSDS White Paper.

Exploration of the Ice Giant Systems.

11. Moore et al. (2020) PSDS White Paper.

Exploration Strategy for the Outer Planets 2023-32.

12. Hendrix, A., Hurford, T. and the ROW Team.

https://www.lpi.usra.edu/opag/ROW/

13. Charnoz, S., et al., 2018. In Planetary Ring

Systems, 517-538.

14. Kollmann et al., 2020. Planetary Science and

Astrobiology Decadal Survey White Paper.

15. Cartwright et al., 2020. Planetary Science and

Astrobiology Decadal Survey White Paper.

16. Ahrens et al., 2020. Planetary Science and

Astrobiology Decadal Survey White Paper.

17. Greenberg, R. et al., 1990. In Uranus, U of A.

18. Beddingfield, C. B., and R. J. Cartwright, 2020.

Icarus 343: 113687.

19. Grundy, W.M. et al., 2006. Icarus 184, 543-555.

20. Moore, M.H., et al., 2007. Icarus, 190, 260-273.

21. Sori, M.M., et al., 2017. Icarus, 290, 1-13.

22. Cartwright, R.J., et al., 2018. Icarus 314, 210-31.

23. Cartwright, Richard J., et al., 2020. Icarus 338.

24. Jankowski, D.G., Squyres, S.W. 1988. Science,

241, 1322-1325.

25. Kargel, J.S. 1995. In Comparative Planetology

with an Earth Perspective.

26. Ćuk, Matija, et al., 2020. arXiv preprint

arXiv:2005.12887.

27. Bauer, J. M., et al., 2002. Icarus 158.1: 178-190.

28. Nicholson, P.D., et al., 2018a. In Planetary Ring

Systems. Cambridge U. Press. 93-111.

29. Porco, C.C., Goldreich, P. 1987a. AJ 93, 724.

30. Porco, C.C., Goldreich, P. 1987b. AJ 93, 730.

31. Braga-Ribas, F. et al. 2014. Nature 508, 72-75.

32. Ortiz, J.L., et al. 2017. Nature 550, 219-223.

33. Ćuk, M. et al., 2016. The Astrophysical Journal

820.2: 9
34. Kirchoff, M.R., et al., 2018. Enceladus and the Icy Moons of Saturn, 267-284.

35. de Kleer, K.R., et al., 2013. DPS.

36. Colwell, J.E., et al., 2018. Icarus 300, 150-166.

37. Tiscareno, M.S. et al. 2019. Science, 364.6445.

38. Horn, L.J., et al. 1988. Icarus 76, 485-492.

39. Chancia, R.O., Hedman, M.M. 2016. AJ 152, 211.

40. Hedman, M.M., Nicholson, P.D. 2014. MNRAS 444, 1369-1388.

41. Hedman, M.M., et al., 2018. In Planetary Ring

Systems. Cambridge U. Press. 308-337.

42. de Pater, I., et al., 2006. Science, 312, 92-94.

43. Showalter, M.R. and Lissauer, J.J., 2006.

Science, 311, 973-977.

44. Denk, T., Mottola, S., 2019. Icarus 322, 80-102. 45. Jewitt, D. and Haghighipour, N., 2007. Annual

Review of Astronomy and Astrophysics, 45.

46. Ness, N.F., et al., 1986. Science 233, 85-9.

47. Connerney, J.E.P., et al., 1987. JGR 92, 15329.

48. Herbert, F., 2009. JGR Sp. Phys. 114, 1-13.

49. Soderlund, K.M., et al., 2013. Icarus 224, 97-113.

50. Stanley, S., Bloxham, J., 2006. Icarus 184, 556-72.

51. Cavazzoni, C., 1999. Science (80). 283, 44-46.

52. Millot, M., et al., 2019. Nature 569, 251-255.

53. Wilson, H.F., et al., 2013. Phys. Rev. Lett. 110.

54. Hofstadter, M., et al., 2017. JPL D-100520

55. Bagenal, F., 1992. Giant planet magnetospheres.

Annu. Rev. Earth Planet. Sci.

56. Cao, X., Paty, C., 2017. JGR Space Physics. 122, 6318-6331.

57. Tóth, G., et al., 2004. JGR Space Physics 109.

58. Glatzmaiers, G.A. and Roberts, P.H., 1995.

Nature 377, 203-209.

59. Cheng, Andrew F., et al., 1987. JGR Space

Physics 92.A13: 15315-15328.

60. Marley, Mark S. Icarus 234 (2014): 194-199.

61. Elder, C. M., et al., 2018. Acta Astro. 148, 1-11.

62. Jarmak, S., et al., 2020. Acta Astro.170: 6-26.

63. Cohen et al., 2020. Planetary Science and

Astrobiology Decadal Survey White Paper.

64. Elder, C. M., et al., 2020. LPI 2326, 2277.

65. Kivelson, M. G., et al., 1999. JGR, 104(A3), 4609- 4626.

66. Zimmer, C., et al., 2000. Icarus, 147, 329-347.

67. Conversano, R.W., 2019. IEEE Aerospace

Conference, Big Sky, MT, USA, pp. 1-8.

68. Vaquero M, et al., 2019. American Astronautical Society Meeting Abstracts, AAS 19-265.

69. Balint, T.S., et al., 2020. Planetary Science and Astrobiology Decadal Survey White Paper. 